\title{
Illumination Compensation and Normalization for Automatic Recognition of Rapid Diagnostic Kits
}

\author{
Jaean Jung1, Won-Jung Kim¹, Byung-Gap Hwang1, Joong-Eun Jung2, Ho-Joon Kim²* \\ 1 The MediSensor, Inc., Daegu Technopark, 711 Hosan-dong, Dalseo-gu, Daegu, Korea. \\ 2 The School of Computer Science and Electrical Engineering, Handong Global University, Pohang, 791-708, \\ Korea. \\ * Corresponding author. Tel.: +820542601373; email: hjkim@handong.edu \\ Manuscript submitted August 10, 2014; accepted March 25, 2015. \\ doi: 10.17706/ijcce.2015.4.4.256-263
}

\begin{abstract}
In this paper, we present an illumination compensation technique and a region of interest (ROI) extraction method for the recognition of medical diagnostic kits. The Hough transform and a multi-stage image segmentation technique are used for the ROI extraction method. To improve the accuracy of quantitative recognition, an illumination normalization method which is adaptive to the variations of background image and lighting conditions is proposed. Varying illumination condition may cause a distortion of color and intensity information of the target image. Therefore, we propose a data conversion method called illumination compensation curve which can be adjusted by a set of reference image patterns. The curve is generated by calculating the compensation factor from the change of average RGB values in the ROI of the reference image patterns. We discuss the usability of the suggested methods through the experimental results which are applied to 20 types of kit image patterns.
\end{abstract}

Key words: Color recognition, diagnostic kits, Hough transform, illumination compensation.

\section{Introduction}

Automatic recognition system for medical diagnostic kits makes it possible to overcome the limitation of recognition by naked eyes in the process of evaluating the color and intensity information from the diagnosing results. Also, it allows pursuing the real-time responsiveness of the system as well as the convenience of the diagnosing process. However, in implementing the system, there are some difficulties such as the change of background image and lighting conditions.

In recent researches, various image representation method insensitive to varying illumination were proposed [1]-[6]. Xie et al. proposed an illumination normalization method for human face recognition in which a face image is treated as a combination of a sequence of facets. In their method, the effect of the illumination on each facet is modeled by a multiplicative noise and an additive noise [1]. Chen et al. proposed a multi-phase scheme to balance uneven light distribution and to produce content with a high degree of recognition of text images [2].

The Hough transform is a common technique for straight line detection. Numerous varieties and applications of line detection had been studied. Ji et al. proposed an improved Hough transform method for robust detection of line segments in images with complicated backgrounds [7]. Tu et al. introduced a method for monitoring the position of a vehicle on a lane [8]. In their research, the correlation of sequential images and the constraints of lane lines and camera are used to minimize the region of interest (ROI) in 
both image space and Hough transform space.

In this paper, we introduce an illumination compensation technique and a ROI segmentation method for the automatic diagnostic kits recognition system. We propose a ROI extraction method which consists of an adaptive background subtraction process and region segmentation process using the Hough transform.

We define a data conversion function called illumination compensation curve which is trained by the reference image pattern data. We introduce the detailed method of illumination compensating for quantitative evaluation of the diagnosing results in the system. From the experimental results, we show that the system using the proposed method is less sensitive to varying illumination conditions.

\section{Backgrounds}

The form of the diagnosis kits concerned in this paper is shown in Fig. 1. As shown in the figure, ROI area of the measurement target is classified as kits peripheral area, diagnosis object area, and color band area which represents reaction degree of reagent. For convenience, we have named these three areas as B1 area, B2 area and B3 area, respectively. In addition, the kit includes the test area and control index that is a reference pattern to judge whether it is defective or not.

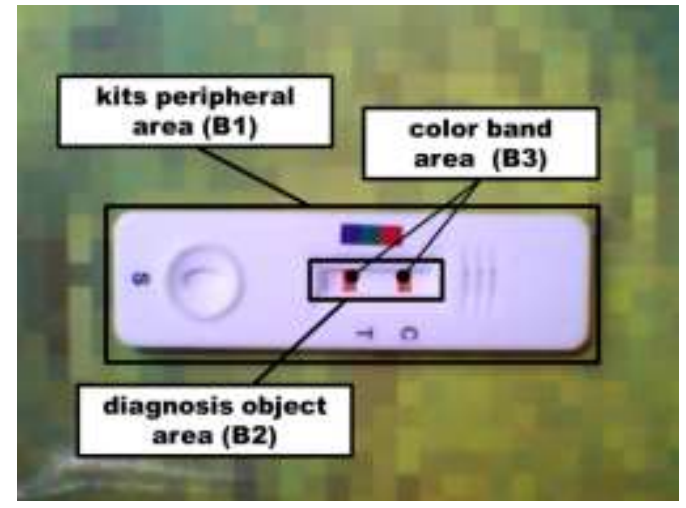

Fig. 1. ROI in the diagnostic kit image.

We have developed two types of system, a PC-based system and a smart phone-based mobile application as shown in Fig. 2.

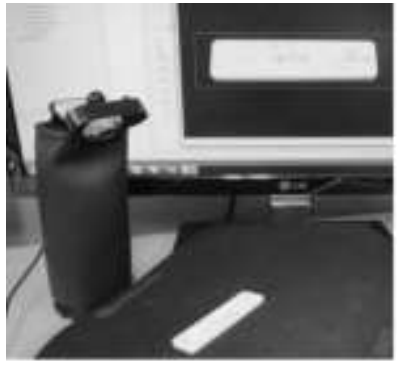

(a)

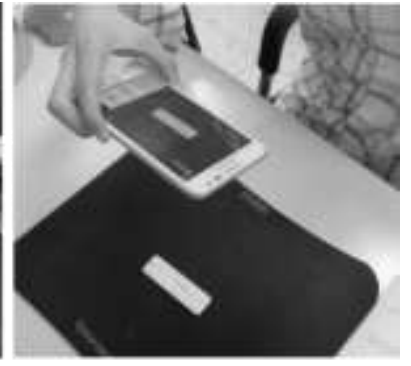

(b)

Fig. 2. Examples of developed system (a) PC-based system and (b) smart phone-based system.

\section{Diagnostic Kit Image Analysis}

Fig. 3 shows the structure of the underlying system. The system consists of four stages: ROI extraction, illumination compensation, qualitative analysis and quantitative analysis. In the ROI extraction stage, three types of regions (B1, B2 and B3) are extracted. The illumination compensation process is to adjust the 
intensity data and the color information so that the evaluation results are insensitive to varying lighting environments. This process is followed by the qualitative and quantitative analysis of the diagnosing image.

For the ROI segmentation, we first extract B1 area by conducting binarization to the input image, then we acquire feature points in the kit area by labeling, and we extract B2 area through analyzing the relations among the feature points. We execute illumination compensation prior to the analyzing of B2 area and the compensated B2 image data go through leveled qualitative analysis process. The qualitative analysis process consists of three step processes, compensated image generation, contour detection and histogram equalization. The quantitative analysis result generates the intensity level and color information of each target region.

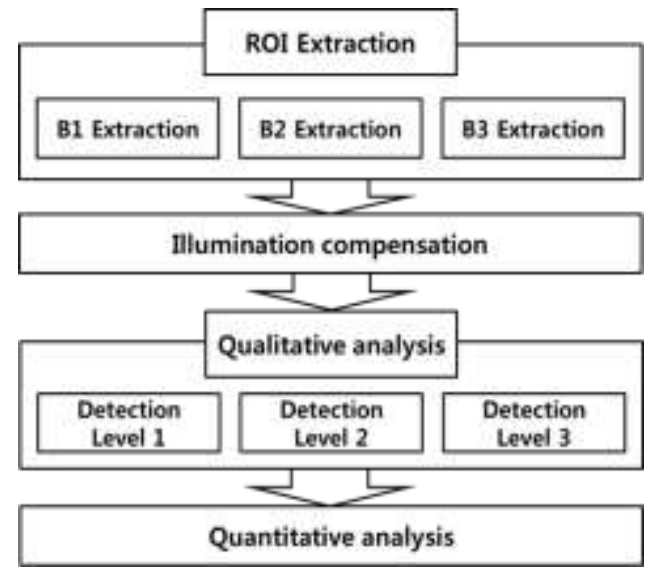

Fig. 3. Diagnostic kit image analysis system.

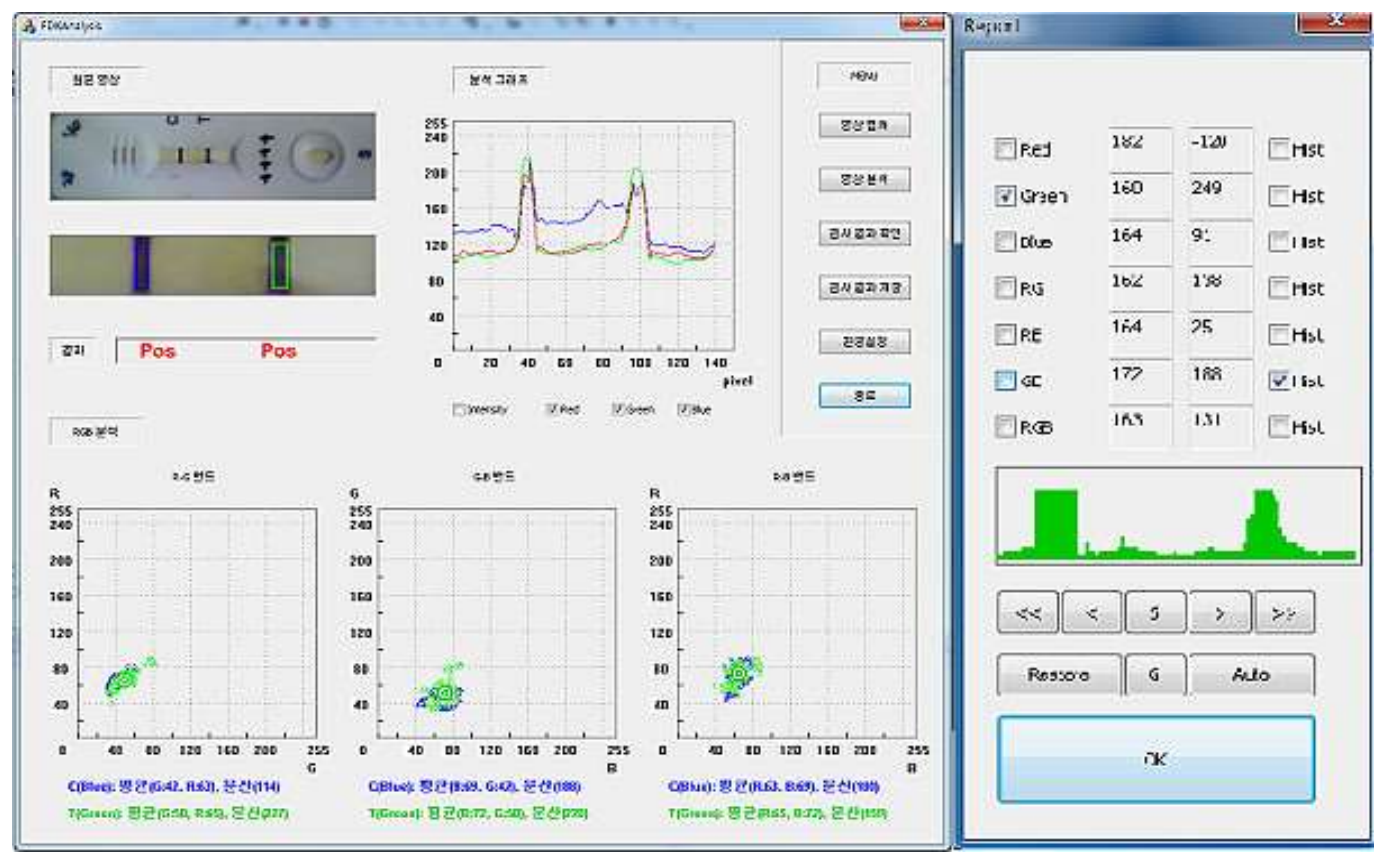

Fig. 4. User interface of the image analysis software.

Fig. 4 shows an illustration of user interface of the developed system. As shown in figure, the display includes the extracted ROI image, target image, and the qualitative and quantitative analysis results. The upper graph shows the RGB value distribution for a horizontal base line. The lower three graphs are two-dimensional graph for each RGB band. This is not only providing an automatic recognition capability, but also supporting the analysis with naked eyes through magnification image of ROI area that is extracted 
from the image. The right part of Fig. 4 shows numerical values for the target image analysis results. We can control the parameter values by using the buttons as shown in the left-lower part of the figure.

\section{ROI Detection Technique}

ROI segmentation process consists of eliminating the background image and extracting the precise object area. Hough transform is used for the ROI detection process. The accuracy of the segmentation may be degraded due to the effect of the illumination changes. Thus, in this paper, we applied a method to update the background image adaptively. Equation (1) shows the update scheme to maintain the background image.

$$
x_{b}^{t+1}(i)=\left\{\begin{array}{cc}
x_{b}^{t}(i) & \text { if } x_{c}^{t}(i) \in x_{i} \\
\alpha\left(x_{b}^{t}(i)\right)+(1-\alpha) x_{c}^{t} & \text { otherwise. }
\end{array}\right.
$$

In the equation, and mean the $i^{\text {th }}$ pixel value of the background image and the current image at time $t$, respectively. In other words, running parallel with recognition process, the gradual changes of the background image can be reflected by updating pixel values of background image through the calculation between weighted value average and previous value. We extract diagnosis kit area using a straight line detection technique which uses Hough transform. In the image space, the straight line can be described as (2), where the parameter $a$ is the slope of the line, and $b$ is the intercept (y-intercept).

$$
y=a x+b
$$

Duda and Hart proposed the use of a different pair of parameters, denoted $\gamma$ and $\theta$, for the lines in the Hough transform. These two values, taken in conjunction, define a polar coordinate. The parameter $\gamma$ represents the algebraic distance between the line and the origin, whereas $\theta$ is the angle of the vector orthogonal to the line and pointing toward the half upper plane. If the line is located above the origin, $\theta$ is simply the angle of the vector from the origin to this closest point.

The line equation is expressed as (3). A line segment in digital images is a set of pixels. According to (3), each pixel is projected to sine curve in the parameter space. Depending on the number of rows and columns in the image, $n_{r}$ and $n_{c^{\prime}}$ the parameters $\rho$ and $\theta$ very inside ranges of $\left[-n_{r}, \sqrt{n_{r}^{2}+n_{c}^{2}}\right]$ and $[0, \pi)$, respectively.

$$
\rho=x \cos \theta+y \sin \theta
$$
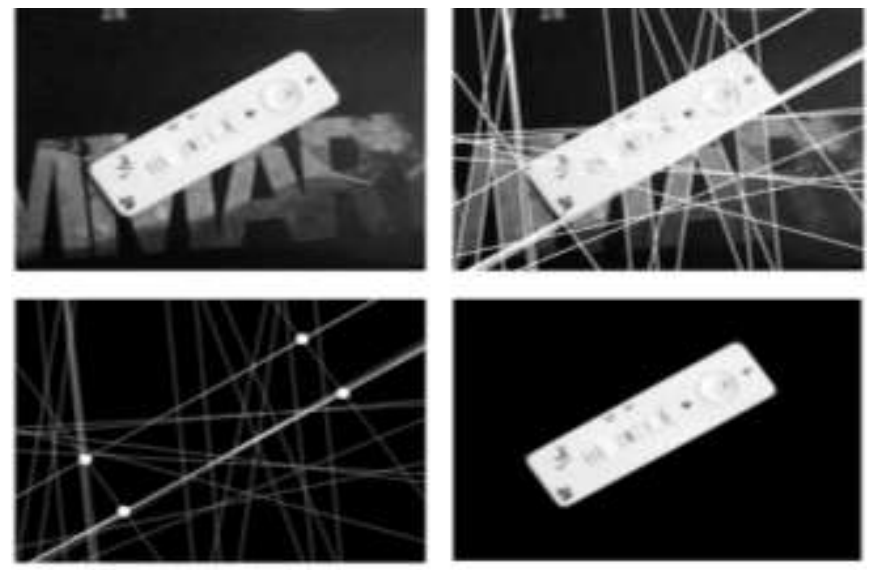

Fig. 5. Intermediate results of the ROI extraction process. 
We can extract the kit area through the matching process which analyzes the interrelationship between straight line and intersection information among the detected line segments. We have shown an example of experiment result applying these processes in Fig. 5.

\section{Illumination Normalization Technique}

We suggest an illumination normalization technique which compensates the RGB color value of the test image from the result that analyzes the relationship between the intensity changes and the RGB color value changes. In order to measure the illumination changes of image, we assume a standard illumination intensity that will be compared to the illumination condition of input image and reference area among the diagnostic kit perimeter (B1). We compose a learning data through sampling in the set of reference patterns which are generated by revising the intensity value by level in illumination environment that controlled to the designated 20 color patterns. From the data, we generate the shape of RGB value depending on the illumination changes like the Fig. 6. From the results, we calculate the compensation factor depending on the illumination change ranges at each color factors. Repeatedly, the difference between measured intensity value and standard intensity in reference area become the factors of multidimensional function which will determine the proportion of RGB compensation value. We can show this multidimensional function to the illumination compensation curve as shown in the figure.

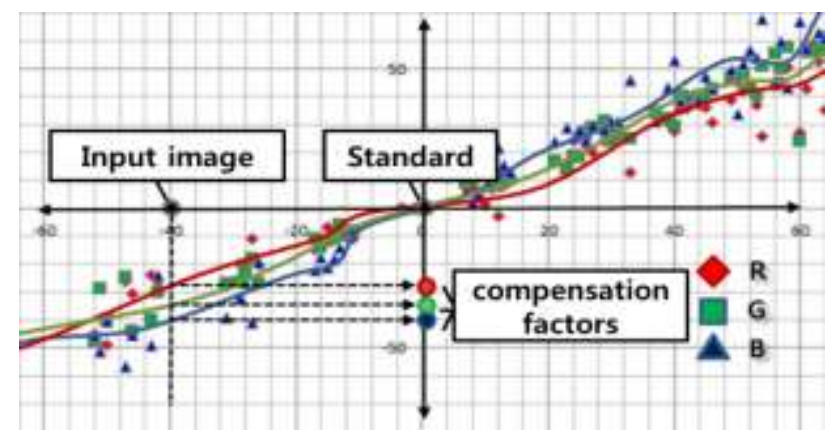

Fig. 6. Illumination compensation curve.

To the area of the measured illumination changes in the reference area of test images, we can obtain compensated color information by calculating proportional value which is relevant to the curve.

The curve in Fig. 6 is represented as a compensation function $f(d)$ which is a function of the deviation at the reference region. The compensation result is determined by (4), where $x^{\prime}$ is the observed value of the test image. To sum up, the color distinction value $x$ is calculated by adding up the observed value $x^{\prime}$ and color compensation function value $f(d)$ for each color band.

$$
x=x^{\prime}+f(d) .
$$

\section{Experiment Results}

We have conducted the experiments using real diagnosis kit images. To generate the illumination compensation curve, we have used 20 kinds of color patterns under five levels of illumination conditions. We have observed the distributions of the intensity and color data for each pattern. Fig. 7 illustrates the recognition results in which the color data for 3 kinds of image patterns are displayed. We have marked the average value measured in standard intensity for the three patterns with double circle. As shown in the figure, the total error of the observed patterns for the standard values can be reduced by the compensation process. 


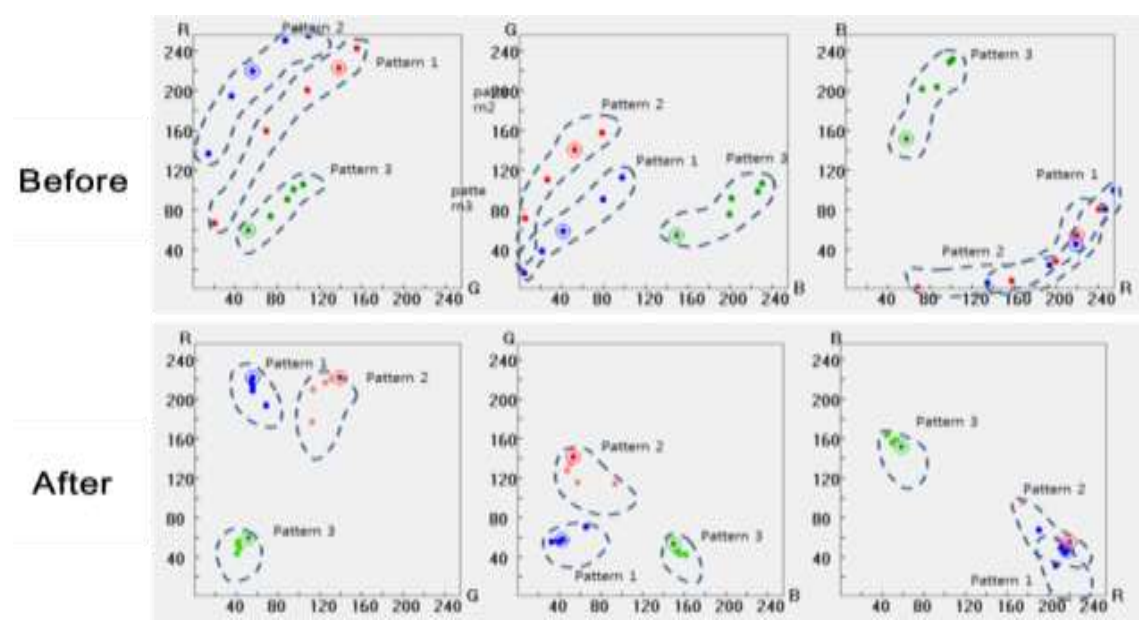

Fig. 7. Color data distribution and standard values.

A total of 216 data that are measured in 12 kinds of environment to 18 kinds of diagnosis kits have been used for the experiments. Among them, 96 patterns have been used for the training, and the rest of the 120 patterns have been used for the test and analysis processes. Fig. 8 shows the recognition performance for arbitrary 4 patterns as a similarity measure. As shown in the figure, the similarities of the recognition results and the reference data for each pattern are better than the results before the compensation process.

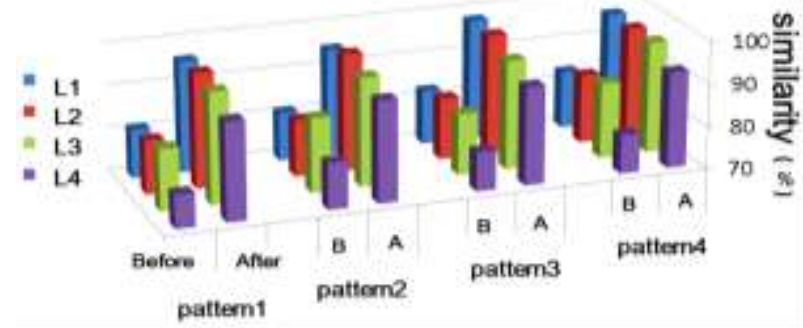

Fig. 8. Comparison of color recognition result.

Table 1 shows the compensated data compared the measured value and the standard data for 10 patterns. The result shows that we can reduce the error rate by applying the illumination compensation curve which is generated by the training process.

Table 1. Compensated, Measured, and Standard Value

\begin{tabular}{lccc}
\hline \hline $\begin{array}{l}\text { Pattern } \\
\text { number }\end{array}$ & Standard data & Measured & Compensated \\
\hline 1 & $(196,046,051)$ & $(242,085,093)$ & $(200,042,035)$ \\
2 & $(052,110,155)$ & $(089,151,196)$ & $(049,117,159)$ \\
3 & $(064,117,080)$ & $(039,076,052)$ & $(074,105,092)$ \\
4 & $(152,170,105)$ & $(109,130,069)$ & $(146,154,107)$ \\
5 & $(046,044,235)$ & $(072,090,253)$ & $(029,050,195)$ \\
6 & $(073,114,239)$ & $(121,164,254)$ & $(083,127,211)$ \\
7 & $(182,011,147)$ & $(132,001,093)$ & $(163,028,127)$ \\
8 & $(193,014,062)$ & $(237,039,110)$ & $(199,003,066)$ \\
9 & $(071,040,088)$ & $(109,077,142)$ & $(068,036,087)$ \\
10 & $(162,151,215)$ & $(207,193,254)$ & $(168,152,201)$ \\
\hline \hline
\end{tabular}

\section{Conclusion}

Due to the variation of illumination condition, the weakness of the automatic image recognition for 
diagnostic kit is the lack of accuracy and preciseness. However, it is very useful technique in response time of the analysis process. The proposed method can minimize the error which might be caused by the variations of background images and illumination environments. Therefore, it can improve the accuracy in the image recognition process for the quantitative analysis as well as the qualitative analysis. The illumination compensation curve is trained by the intensity and color data of the reference area and the test area in the learning pattern images. The suggested method improves the reliability of diagnosis by overcoming several constraints such as the object location in test image, the changes of background image, the illumination condition and the distance between the camera and target objects.

\section{Acknowledgment}

This research was financially supported by the Ministry of Education (MOE) and National Research Foundation of Korea (NRF) through the Human Resource Training Project for Regional Innovation (No. 2012H1B8A2025800)

\section{References}

[1] Xie, X. D., \& Lam, K.-M. (2006). An efficient illumination normalization method for face recognition. Pattern Recognition Letters, 27, 609-617.

[2] Chen, K.-N., Chen, C.-H., \& Chang, C-C. (2012). Efficient illumination compensation techniques for text images. Digital Signal Processing, 22, 726-733.

[3] Lian, Z. C., Er, M. J., \& Liang, Y. C. (2012). A novel efficient local illumination compensation method based on DCT in logarithm domain. Pattern Recognition Letters, 33, 1725-1733.

[4] Lee, D.-S., Ko, M.-S., Seo, Y.-H., et al. (2013). Illumination compensation for multi-view video based on layered histogram matching with depth information. Optics Communications, 286, 74-84.

[5] Wang, C., \& Li, Y. P. (2010). Combine image quality fusion and illumination compensation for video-based face recognition. Neurocomputing, 73, 1478-1490.

[6] Han, H., Shan, S. G., Chen, X. L., \& Gao, W. (2013). A comparative study on illumination preprocessing in face recognition. Pattern Recognition, 46, 1691-1699.

[7] Ji, J. H., Chen, G. D., \& Sun, L. N. (2011). A novel Hough transform method for line detection by enhancing accumulator array. Pattern Recognition Letters, 32, 1503-1510.

[8] Tu, C., Wyk, B. J. van, Hamam, Y., Djouani, K., \& Du, S. Z. (2013). Vehicle position monitoring using Hough transform. IERI Procedia, 4, 315-322.

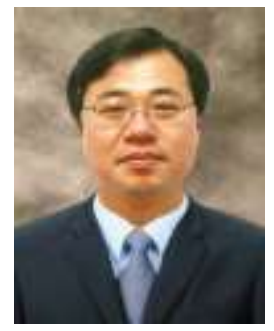

Jaean Jung received his M.S. degree in biochemistry/molecular biology from the Agricultural Chemistry Dept., Korea University, in 1991. He is currently the CEO of Medisensor. Inc., Korea. His research interests include the development of immune and molecular diagnostics kit involving infectious and cardiovascular diseases etc.

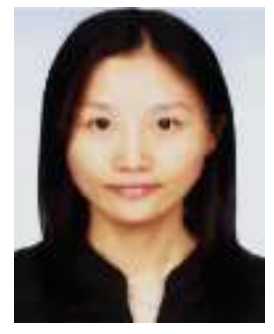

Won-Jung Kim received her Ph.D. degree in molecular immunology from Kyungpook National University, Korea in 2010. She is currently a principle researcher at Medisensor, Inc., in Korea. Her research is interested in the development of immune and molecular diagnostics kit involving infectious and cardiovascular diseases etc. 


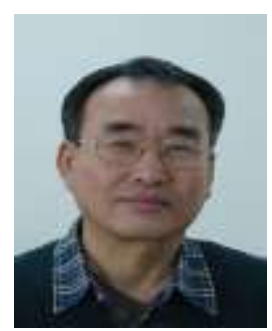

Byung-Gap Hwang received his Ph.D. degree in biochemistry/molecular biology from the Agricultural Chemistry Dept., Korea University, in 1994. He is currently the research institute director of Medisensor. Inc., Korea. His research is focused on the areas in the development of immune and molecular diagnostics kit involving infectious and cardiovascular diseases etc.

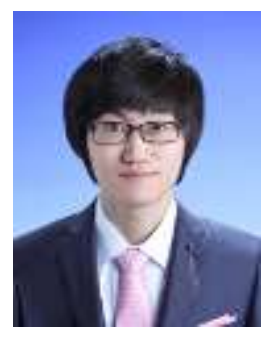

Joong-Eun Jung received a bachelor's degree of in computer engineering from Handong Global University, Pohang, Kyeongbuk, Korea in 2013. He is currently studying for his master's course in Handong Global University. He is knowledgeable in computer vision, artificial intelligence and mobile programming.

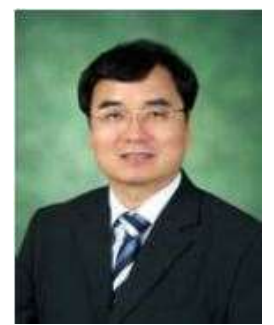

Ho-Joon Kim received the B.S. degree in computer engineering from Kyeongbuk National University, Korea, in 1987 and the Ph.D. degree in computer science from Korea Advanced Institute of Science and Technology (KAIST) in 1995. He worked as a researcher at the Korea Atomic Energy Research Institute from 1987 to 1991. Currently he is a professor at the School of Computer Science and Electric Engineering, Handong Global University, Korea.

His research interests include machine vision, pattern recognition, neural network architectures, and medical image processing.

Prof. Kim received the Best Paper Award from the technical program committee of IEEE International Conference on Neural Networks and Signal Processing in 2008. 